ANNALES

POLONICI MATHEMATICI

$99.2(2010)$

\title{
Rectilinearization of functions definable by a Weierstrass system and its applications
}

\author{
by KrzYsZTof Jan NowaK (Kraków)
}

\begin{abstract}
This paper presents several theorems on the rectilinearization of functions definable by a convergent Weierstrass system, as well as their applications to decomposition into special cubes and quantifier elimination.
\end{abstract}

1. Introduction. This paper is concerned with the rectilinearization of functions definable in the expansion $\mathcal{R}$ of the real field with restricted analytic functions determined by a given convergent Weierstrass system. We prove several theorems on this subject, including counterparts of the ones on rectilinearization of a continuous subanalytic function due to BierstoneMilman [1] and Parusiński [16.

In the real analytic case, Bierstone and Milman proved that if $U$ is a real analytic manifold and $f: U \rightarrow \mathbb{R}$ is a continuous subanalytic function, then there exists a locally finite covering $\left(\pi_{j}: U_{j} \rightarrow U\right)_{j}$ such that:

(i) each $\pi_{j}$ is a composite of finitely many mappings each of which is either a local blowing-up with smooth center or a local power substitution;

(ii) each $f \circ \pi_{j}$ is analytic.

In the proof, they employ Hironaka's major complex-analytic tool, the local flattening theorem (see e.g. 8]), and reduce the problem to the case where the subanalytic function $f$ under study satisfies a non-trivial analytic equation. Then, via transformation to normal crossings, the Weierstrass preparation theorem can be applied to that equation. This leads to the case where the function $f$ is a root of a polynomial with analytic coefficients.

Parusiński, in turn, applying a real-analytic version of the AbhyankarJung theorem, improved the above result to show that the mappings $\pi_{j}$ can

2010 Mathematics Subject Classification: Primary 32S45, 32B20; Secondary 14P15, 03C10. Key words and phrases: rectilinearization, subanalytic functions, transformation to normal crossings, fractional normal crossings, special cubes and modifications, quantifier elimination. 
be finite composites of local blowings-up and and power substitution only at the last step.

In our approach to the subject, we make use of a characterization of an $\mathcal{R}$-definable function by terms in the language augmented by the names of the reciprocal function and roots. This characterization follows, through a classical model-theoretic argument, namely, a theorem of Herbrand, from the fact that, in the augmented language, the structure under study admits quantifier elimination and has a universal axiomatization. The latter is a generalization of the classical theorem about the structure $\mathbb{R}_{\text {an }}$ due to van den Dries-Macintyre-Marker [7]. They, in turn, applied quantifier elimination for the structure $\mathbb{R}_{a n}$, established by Denef-van den Dries [4. For the proofs of those results through Robinson's test, see also [10].

The aforementioned proofs of those classical results rely essentially on the Weierstrass preparation theorem, and thence on good algebraic properties of the local rings of real convergent power series (like, for instance, noetherianity and flatness). They can thus be adapted - in particular, the one based on Robinson's test from our paper [10] - to the case of real convergent Weierstrass systems. Note that we cannot attain here the description of definable functions by the methods developed in our paper [12, because we are not able to decompose germs from a given Weierstrass system according to certain diagonal splitting of their Taylor coefficients.

Several theorems concerning rectilinearization of quasi-subanalytic functions are provided in Section 2. These results will be applied in the next section and in our subsequent papers [14, 15] as well. Section 3 offers an affirmative answer, for the case of the structure $\mathcal{R}$, to a problem posed in our previous article [11] concerning the decomposition of a definable set into special cubes. Consequently, we achieve the quantifier elimination for $\mathcal{R}$. This generalizes the result of Denef-van den Dries [4] for the structure $\mathcal{R}_{a n}$.

Now, we turn to the notion of a Weierstrass system, introduced by DenefLipshitz [5] in connection with their generalizations of Artin's approximation theorems. Convergent Weierstrass systems were investigated by van den Dries [6]. We now recall the precise definitions. By a Weierstrass system over the field $\mathbb{K}=\mathbb{R}$ or $\mathbb{K}=\mathbb{C}$, we mean a collection $\mathcal{W}=\left(\mathcal{W}_{n}\right)_{n \in \mathbb{N}}$ of $\mathbb{K}$-subalgebras of $\mathbb{K}\left[\left[x_{1}, \ldots, x_{n}\right]\right]$ that satisfy the following conditions:

1. $\mathbb{K}\left[x_{1}, \ldots, x_{n}\right] \subset \mathcal{W}_{n}, \mathcal{W}_{n+1} \cap \mathbb{K}\left[\left[x_{1}, \ldots, x_{n}\right]\right]=\mathcal{W}_{n}$ and $\mathcal{W}_{n}$ is closed under permutation of variables;

2. $\mathcal{W}_{n}$ is closed under reciprocal;

3. $\mathcal{W}_{n}$ is closed under Weierstrass division.

Property 2 implies that each $\mathcal{W}_{n}$ is a local ring. It follows easily from the Weierstrass division that the rings $\mathcal{W}_{n}$ are closed under substitution, division by a coordinate, differentiation, inverse and implicit functions. The unique 
maximal ideal of $\mathcal{W}_{n}$ is generated by the coordinates $x_{1}, \ldots, x_{n}$. Moreover, every local ring $\mathcal{W}_{n}$ is henselian, noetherian, regular and excellent (by virtue of a Jacobian criterion for a ring to be excellent, cf. [9, Section 40, Theorem 102], and its completion is $\mathbb{K}\left[\left[x_{1}, \ldots, x_{n}\right]\right]$. Thus, from the algebraic viewpoint, the rings $\mathcal{W}_{n}$ possess the best possible properties.

We call a Weierstrass system $\left(\mathcal{W}_{n}\right)_{n \in \mathbb{N}}$ convergent if, for all $n \in \mathbb{N}, \mathcal{W}_{n} \subset$ $\mathbb{K}\left\{x_{1}, \ldots, x_{n}\right\}$ and $\mathcal{W}_{n}$ is closed under analytic prolongation. An analytic function germ $f\left(x_{1}, \ldots, x_{n}\right) \in \mathcal{W}_{n}$ is said to be $\mathcal{W}$-convergent or a $\mathcal{W}$-germ. If $U \subset \mathbb{K}^{m}$ is an open subset, an analytic function $f: U \rightarrow \mathbb{K}$ is called a $\mathcal{W}$-analytic function if all its germs are $\mathcal{W}$-convergent. As in the classical analytic geometry, this gives rise to the category $\mathcal{W}$ of $\mathcal{W}$-analytic manifolds, $\mathcal{W}$-analytic, $\mathcal{W}$-semianalytic or $\mathcal{W}$-subanalytic sets as well as $\mathcal{W}$-analytic, $\mathcal{W}$-semianalytic or $\mathcal{W}$-subanalytic functions.

It is often convenient to write down analytic germs together with the variables, and therefore we use the following notation:

$$
\mathcal{W}_{n}=: \mathbb{K}\left\langle x_{1}, \ldots, x_{n}\right\rangle \subset \mathbb{K}\left\{x_{1}, \ldots, x_{n}\right\} .
$$

From now on, we fix a real convergent Weierstrass system $\mathcal{W}$ (over $\mathbb{R}$ ). Consider the expansion $\mathcal{R}$ of the real field $\mathbb{R}$ by restricted $\mathcal{W}$-analytic functions, i.e. functions of the form

$$
\tilde{f}(x)= \begin{cases}f(x) & \text { if } x \in[-1,1]^{m} \\ 0 & \text { otherwise }\end{cases}
$$

where $f(x)$ is a $\mathcal{W}$-analytic function in the vicinity of the compact cube $[-1,1]^{m}$. We adjoin to the language of ordered rings (with the symbols $=,<$, $0,1,+,-, \cdot)$ the names of all restricted $\mathcal{W}$-analytic functions.

$\mathcal{R}$ is a polynomially bounded o-minimal structure which admits $\mathcal{W}$ analytic cell decomposition (cf. [17, 11, 12]). As already mentioned, $\mathcal{R}$ admits quantifier elimination and has a universal axiomatization in the language of restricted $\mathcal{W}$-analytic functions augmented by the names of the reciprocal function and roots. Here we describe the reciprocal function $1 / x$ and roots $\sqrt[n]{x}$ in the ordinary fashion by stipulating that:

$$
\begin{array}{lllll}
x \cdot 1 / x=1 & \text { if } x \neq 0 \quad \text { and } & 1 / x=0 & \text { if } x=0, \\
(\sqrt[n]{x})^{n}=x & \text { if } x \geq 0 & \text { and } & \sqrt[n]{x}=0 & \text { if } x<0 .
\end{array}
$$

Below, the word "definable" will mean "definable in the structure $\mathcal{R}$ ".

2. Rectilinearization of quasi-subanalytic functions. We begin with some terminology suitable for rectilinearization of definable functions. By a quadrant in $\mathbb{R}^{m}$ we mean a subset of $\mathbb{R}^{m}$ of the form

$\left\{x=\left(x_{1}, \ldots, x_{m}\right) \in \mathbb{R}^{m}: x_{i}=0, x_{j}>0, x_{k}<0\right.$ for $\left.i \in I_{0}, j \in I_{+}, k \in I_{-}\right\}$, 
where $\left\{I_{0}, I_{+}, I_{-}\right\}$is a disjoint partition of $\{1, \ldots, m\}$; its trace $Q$ on the cube $[-1,1]^{m}$ will be called a bounded quadrant; put

$$
Q_{+}:=\left\{x \in[0,1]^{m}: x_{i}=0, x_{j}>0 \text { for } i \in I_{0}, j \in I_{+} \cup I_{-}\right\} .
$$

The interior $\operatorname{Int}(Q)$ of the quadrant $Q$ is its trace on the open cube $(-1,1)^{m}$. A bounded closed quadrant is the closure $\bar{Q}$ of a bounded quadrant $Q$, i.e. a subset of $\mathbb{R}^{m}$ of the form

$$
\bar{Q}:=\left\{x \in[-1,1]^{m}: x_{i}=0, x_{j} \geq 0, x_{k} \leq 0 \text { for } i \in I_{0}, j \in I_{+}, k \in I_{-}\right\} .
$$

We say that a function $g$ on a bounded quadrant $Q$ in $\mathbb{R}^{m}$ is a fractional normal crossing on $Q$ if it is the superposition of a normal crossing $f$ in the vicinity of $\overline{Q_{+}}$and a rational power substitution $\psi$ given by the equality

$$
\psi: \mathbb{R}^{m} \rightarrow \mathbb{R}^{m}, \quad \psi\left(x_{1}, \ldots, x_{m}\right)=\left(\left|x_{1}\right|^{\alpha_{1}}, \ldots,\left|x_{m}\right|^{\alpha_{m}}\right),
$$

where $\alpha_{1}, \ldots, \alpha_{m}$ are non-negative rational numbers. In other words, a fractional normal crossing $g$ on $Q$ is a function of the form

$$
g\left(x_{1}, \ldots, x_{m}\right)=\left|x_{1}\right|^{n_{1} / N} \cdot \ldots \cdot\left|x_{m}\right|^{n_{m} / N} \cdot u\left(\left|x_{1}\right|^{1 / N}, \ldots,\left|x_{m}\right|^{1 / N}\right),
$$

where $N$ is a positive integer, $n_{1}, \ldots, n_{m}$ are non-negative integers such that $n_{i}=0$ for $i \in I_{0}$, and $u$ is a $\mathcal{W}$-analytic function near $\overline{Q_{+}}$which vanishes nowhere on $\overline{Q_{+}}$.

Before proving the main result of this section, we make a key observation. Consider an o-minimal expansion $\mathcal{S}$ of the real field in a language $\mathcal{L}$. Since every o-minimal structure has definable Skolem (choice) functions, the following two conditions are equivalent:

- every definable function $f$ in $\mathcal{S}$ is piecewise given by a finite number of terms in the language $\mathcal{L}$;

- the structure $\mathcal{S}$ has a universal axiomatization which admits quantifier elimination in the language $\mathcal{L}$.

In the case of the structure $\mathcal{R}$, every definable function is actually given by one term in the language of restricted $\mathcal{W}$-analytic functions augmented by the names of rational powers. Indeed, every such function is piecewise given by a finite number of terms in this augmented language, and therefore it is sufficient to show that the characteristic function of any definable subset $E \subset \mathbb{R}^{m}$ is given by one term.

Further, due to quantifier elimination, we are reduced to sets described by atomic formulae, and next, since our language contains only two relation symbols $=$ and $<$, to the sets given by the formulae $t(x)=0$ or $t(x)>0$ with any term $t(x)$. Consequently, we have only to know that the characteristic functions $f$ and $g$ of the subsets $A:=\{0\}$ and $B:=(0, \infty)$ of the real line 
$\mathbb{R}$ are given by one term. But this follows from the obvious equalities below:

$$
f(x)=1-x \cdot \frac{1}{x} \quad \text { and } \quad g(x)=x \cdot \frac{1}{(\sqrt{x})^{2}} .
$$

THEOREM 1 (on simultaneous rectilinearization of definable functions). If $f_{1}, \ldots, f_{s}: \mathbb{R}^{m} \rightarrow \mathbb{R}$ are definable functions and $K$ is a compact subset of $\mathbb{R}^{m}$, then there exists a finite collection of modifications

$$
\varphi_{i}:[-1,1]^{m} \rightarrow \mathbb{R}^{m}, \quad i=1, \ldots, p,
$$

such that

1) each $\varphi_{i}$ extends to a $\mathcal{W}$-analytic mapping in a neighbourhood of the cube $[-1,1]^{m}$, which is a composite of finitely many local blowings-up with smooth centers and power substitutions;

2) the union of the images $\varphi_{i}\left((-1,1)^{m}\right), i=1, \ldots, p$, is a neighbourhood of $K$;

3) for every bounded quadrant $Q_{j}, j=1, \ldots, 3^{m}$, the restriction to $Q_{j}$ of each function $f_{k} \circ \varphi_{i}, k=1, \ldots, s, i=1, \ldots, p$, either vanishes or is a normal crossing or a reciprocal normal crossing on $Q_{j}$.

The proof is based on the fact that every definable function $f_{k}: \mathbb{R}^{m} \rightarrow \mathbb{R}$ is piecewise given by one term $t_{k}$ in the language of restricted $\mathcal{W}$-analytic functions augmented by the names of rational powers. We shall proceed by induction on the complexity of the terms $t_{k}$; obviously, terms of complexity zero are variables and constants. We wish to explain the induction process more precisely.

For any finite collection $\left(\psi_{i}\right)$ of modifications described in Theorem 1, denote by $\psi$ the modification being the disjoint gluing of the mappings $\psi_{i}$. In fact, we shall prove by a double induction, with respect to the maximum $n=0,1,2, \ldots$ of the complexities of the terms $t_{k}$ and the number $s=1,2, \ldots$ of these terms, that the theorem holds for the superpositions $t_{k}$ of the terms $t_{k}$ with any modification $\psi$ as above.

When $n=0$ and $s$ is an arbitrary positive integer, the theorem can be established directly via a simultaneous transformation to normal crossings of the components of the mapping $\psi$. We encounter two distinct induction steps, described by the following two schemes:

I. assuming the theorem to hold for $n$ and $s$, we will prove it for $n$ and $s+1$

II. assuming the theorem to hold for $n$ and all $s$, we will prove it for $n+1$ and 1.

We first outline how to cope with induction scheme I. Suppose we have terms $t_{1}, \ldots, t_{s}, t_{s+1}$. By induction hypothesis, we are able to find a finite collection $\varphi$ of modifications such that the requirements of the theorem are 
fulfilled for the superpositions $t_{1} \circ \psi \circ \varphi, \ldots, t_{s} \circ \psi \circ \varphi$. We shall have established this induction scheme if we find a collection $\chi$ of modifications that improves $t_{s+1} \circ \psi \circ \varphi$ without spoiling the superpositions already achieved in an appropriate form. Below, we shall explain how to find such an appropriate collection $\chi$.

Take a collection $\omega$ of modifications suitable for $t_{s+1} \circ \psi \circ \varphi(x)$; let $x=$ $\omega\left(x^{\prime}\right)$. Next, via simultaneous transformation to normal crossings, one can find a collection $\sigma$ of modifications $x^{\prime}=\sigma\left(x^{\prime \prime}\right)$ such that each

$$
x_{j}=\omega_{j}\left(x^{\prime}\right)=\left(\omega_{j} \circ \sigma\right)\left(x^{\prime \prime}\right) \quad \text { and } \quad x_{j}^{\prime}=\sigma_{j}\left(x^{\prime \prime}\right), \quad j=1, \ldots, m,
$$

is a normal crossing in the variables $x^{\prime \prime}$. Then the superposition $\psi \circ \varphi \circ \omega \circ \sigma$ is the desired collection of modifications.

In order to establish induction scheme II, we must analyze a term $t$ of complexity $n+1$, and thus encounter the following cases:

$$
t=\sqrt[p]{t_{1}}, \quad t=t_{1} \cdot t_{2}, \quad t=\frac{t_{1}}{t_{2}}, \quad t=t_{1}+t_{2} \quad \text { and } \quad t=g\left(t_{1}, \ldots, t_{r}\right),
$$

where $t_{1}, \ldots, t_{r}$ are terms of complexity $\leq n$ and $g$ is a restricted $\mathcal{W}$-analytic function. The verification of these five cases is routine, and needs again the use of simultaneous transformation to normal crossings of a finite number of $\mathcal{W}$-analytic functions in the following strengthened form: one can require that the exponents of the normal crossings achieved in the process be totally ordered with respect to the induced partial ordering from $\mathbb{N}^{m}$. This finishes the proof, the details being left to the reader.

REMARK. In the above proof, we use the obvious fact that normal crossings are preserved under substitution of powers, and thus under substitutions of normal crossings. Similarly, fractional normal crossings are preserved under substitution of fractional normal crossings.

We say that a real convergent Weierstrass system $\left(\mathcal{W}_{n}\right)_{n \in \mathbb{N}}$ over $\mathbb{R}$ is closed under complexification if the complexification of each germ $f \in \mathcal{W}_{n}$ belongs to the ring $\mathcal{W}_{2 n} \otimes_{\mathbb{R}} \mathbb{C}$; here, we identify the complex space $\mathbb{C}_{z}^{n}$ with the real space $\mathbb{R}_{x, y}^{2 n}$, where $z_{i}=x_{i}+y_{i} \sqrt{-1}$. A complex convergent Weierstrass system $\left(\mathcal{W}_{n}\right)_{n \in \mathbb{N}}$ over $\mathbb{C}$ is closed under taking real and imaginary parts if for each germ

$$
f=\sum_{\alpha \in \mathbb{N}^{n}} c_{\alpha} x^{\alpha} \in \mathcal{W}_{n} \quad \text { with } \quad c_{\alpha}=a_{\alpha}+b_{\alpha} \cdot \sqrt{-1} \in \mathbb{C},
$$

the germs $\sum_{\alpha \in \mathbb{N}^{n}} a_{\alpha} x^{\alpha}, \sum_{\alpha \in \mathbb{N}^{n}} b_{\alpha} x^{\alpha} \in \mathcal{W}_{n}$ belong to the ring $\mathcal{W}_{n}$ too.

Obviously, there is a one-to-one correspondence between the convergent Weierstrass systems of these two types. It is easy to check that the complexification $\mathcal{W}_{n}^{\mathbb{C}}$ of $\mathcal{W}_{n}$ coincides with the following intersection:

(*) $\mathcal{W}_{n}^{\mathbb{C}}\left\langle z_{1}, \ldots, z_{n}\right\rangle=\left(\mathcal{W}_{2 n}\left\langle x_{1}, \ldots, x_{n}, y_{1}, \ldots, y_{n}\right\rangle \otimes_{\mathbb{R}} \mathbb{C}\right) \cap \mathbb{C}\left[\left[z_{1}, \ldots, z_{n}\right]\right]$. 
If the real convergent Weierstrass system $\mathcal{W}$ under study is closed under complexification, we can repeat mutatis mutandis the above proof in order to get modifications $\varphi_{i}$ that are finite composites of local blowings-up and of power substitution only at the last step, as stated below.

TheOREM $1^{*}$ (on simultaneous rectilinearization of definable functions) $\left({ }^{1}\right)$. If $f_{1}, \ldots, f_{s}: \mathbb{R}^{m} \rightarrow \mathbb{R}$ are definable functions and $K$ is a compact subset of $\mathbb{R}^{m}$, then there exists a finite collection of modifications

$$
\varphi_{i}:[-1,1]^{m} \rightarrow \mathbb{R}^{m}, \quad i=1, \ldots, p,
$$

such that

1) each $\varphi_{i}$ extends to a $\mathcal{W}$-analytic mapping in a neighbourhood of the cube $[-1,1]^{m}$, which is a composite of finitely many local blowings-up with smooth centers;

2) the union of the images $\varphi_{i}\left((-1,1)^{m}\right), i=1, \ldots, p$, is a neighbourhood of $K$;

3) for every bounded quadrant $Q_{j}, j=1, \ldots, 3^{m}$, the restriction to $Q_{j}$ of each function $f_{k} \circ \varphi_{i}, k=1, \ldots, s, i=1, \ldots, p$, either vanishes or is a fractional normal crossing or a reciprocal fractional normal crossing on $Q_{j}$.

The only additional difficulty in the proof of Theorem $1^{*}$ is to show induction scheme II for the terms

$$
t=t_{1}+t_{2} \text { and } t=g\left(t_{1}, \ldots, t_{r}\right) .
$$

This will be easily done, once we have at our disposal the following result about fractional normal crossings:

Lemma. Given a $\mathcal{W}$-analytic function $f: U \rightarrow \mathbb{R}$ near zero and $n \in \mathbb{N}$, there exist a finite number of modifications $\sigma_{i}:[0,1]^{m} \rightarrow \mathbb{R}$ such that

(i) each $\sigma_{i}$ extends to a $\mathcal{W}$-analytic mapping in a neighbourhood of the cube $[0,1]^{m}$, which is a composite of finitely many local blowings-up with smooth centers;

(ii) $\sigma_{i}\left([0,1]^{m}\right) \subset U$ and the union of the images $\sigma_{i}\left((0,1)^{m}\right)$ is the trace of a neighbourhood of zero on the orthant $(0, \infty)^{m}$;

(iii) each superposition $f\left(x_{1}^{1 / n}, \ldots, x_{m}^{1 / n}\right) \circ \sigma_{i}$ is a fractional normal crossing on the orthant $(0,1)^{m}$.

In this case, similarly to the case of real analytic functions, the lemma follows from the Abhyankar-Jung theorem and the fact that $f\left(x_{1}^{1 / n}, \ldots, x_{m}^{1 / n}\right)$ is integral over the ring of $\mathcal{W}$-analytic function germs. We can outline the proof as follows.

$\left({ }^{1}\right)$ Here the superscript ${ }^{*}$ indicates that the conclusion holds true provided that the real convergent Weierstrass system under consideration is closed under complexification. 
First, the Abhyankar-Jung theorem remains valid - as proven in our paper [13] - for any excellent henselian ring whose completion is $\mathbb{C}\left[\left[x_{1}, \ldots, x_{m}\right]\right]$. In particular, it is valid for the ring $\mathbb{C}\left\langle x_{1}, \ldots, x_{m}\right\rangle$. Next, the latter assertion can be deduced by complexification, in the same manner as in the classical case, whenever the real Weierstrass system $\mathcal{W}$ under study is closed under complexification. Indeed, the polynomial

$$
P(x ; t):=\prod_{k_{1}, \ldots, k_{m}=0}^{n-1}\left(t-f\left(\varepsilon^{k_{1}} x_{1}, \ldots, \varepsilon^{k_{m}} x_{m}\right)\right),
$$

where $\varepsilon$ is a primitive root of unity, has coefficients which are real analytic functions belonging to

$$
\mathbb{R}\left\langle x_{1}, \ldots, x_{m}\right\rangle \cap \mathbb{R}\left\{x_{1}^{n}, \ldots, x_{m}^{n}\right\}=\mathbb{R}\left\langle x_{1}^{n}, \ldots, x_{m}^{n}\right\rangle ;
$$

the above equality holds because the Weierstrass system $\mathbb{R}\langle x\rangle$ is convergent, and thus closed under analytic prolongation. Hence the assertion follows.

Consequently, $f\left(x_{1}^{1 / n}, \ldots, x_{m}^{1 / n}\right)$ is, after a suitable transformation $\sigma$ to normal crossings by blowing up of the coefficients of the polynomial $P(x ; t)$, a root of a quasiordinary polynomial with free term being a normal crossing. Hence and by the Abhyankar-Jung theorem, $f\left(x_{1}^{1 / n}, \ldots, x_{m}^{1 / n}\right) \circ \sigma$ is a fractional normal crossing, concluding our demonstration of the lemma.

Now let us proceed with some consequences of Theorem 1. Let $U$ be a definable bounded open subset in $\mathbb{R}^{m}, \partial U$ be its frontier and $\rho_{1}, \rho_{2}$ be the distance functions from the sets $U, \partial U$, respectively. Given a definable function $f: U \rightarrow \mathbb{R}$, we can deduce directly from Theorem 1 , applied to the functions $f, \rho_{1}, \rho_{2}$, the following consequence:

THEOREM 2 (on rectilinearization of a definable function). Let $U \subset \mathbb{R}^{m}$ be a bounded open subset and $f: U \rightarrow \mathbb{R}$ be a definable function. Then there exists a finite collection of modifications

such that

$$
\varphi_{i}:[-1,1]^{m} \rightarrow \mathbb{R}^{m}, \quad i=1, \ldots, p,
$$

1) each $\varphi_{i}$ extends to a $\mathcal{W}$-analytic mapping in a neighbourhood of the cube $[-1,1]^{m}$, which is a composite of finitely many local blowings-up with smooth centers and power substitutions;

2) each set $\varphi_{i}^{-1}(U)$ is a finite union of bounded quadrants in $\mathbb{R}^{m}$;

3) each set $\varphi_{i}^{-1}(\partial U)$ is a finite union of bounded closed quadrants in $\mathbb{R}^{m}$ of dimension $m-1$;

4) $U$ is the union of the images $\varphi_{i}(\operatorname{Int}(Q))$ with $Q$ ranging over the bounded quadrants contained in $\varphi_{i}^{-1}(U), i=1, \ldots, p$;

5) for every bounded quadrant $Q$, the restriction to $Q$ of each function $f \circ \varphi_{i}$ either vanishes or is a normal crossing or a reciprocal normal crossing on $Q$, unless $\varphi_{i}^{-1}(U) \cap Q=\emptyset$. 
Remark. One can formulate Theorem 2, similarly to Theorem 1, for several definable functions $f_{1}, \ldots, f_{s}$.

It follows from points 1) and 2) that every bounded quadrant of dimension $<m$ contained in $\varphi_{i}^{-1}(U)$ is adjacent to a bounded quadrant of dimension $m$ (a bounded orthant) contained in $\varphi_{i}^{-1}(U)$. Hence

$$
\varphi_{i}^{-1}(\bar{U})=\overline{\varphi_{i}^{-1}(U)}
$$

and therefore point 4) implies that $\bar{U}$ is the union of the images $\varphi_{i}(\bar{Q})$ of the closures of those bounded quadrants of dimension $m$ (bounded orthants) $Q$ for which $\varphi_{i}(Q) \subset U, i=1, \ldots, p$.

For a bounded orthant $Q$ contained in $\varphi_{i}^{-1}(U)$, denote by $\operatorname{dom}_{i}(Q)$ the union of $Q$ and all those bounded quadrants that are adjacent to $Q$ and disjoint from $\varphi_{i}^{-1}(\partial U)$; it is, of course, an open subset of the closure $\bar{Q}$. Moreover, the open subset $\varphi_{i}^{-1}(U)$ of the cube $[-1,1]^{m}$ coincides with the union of $\operatorname{dom}_{i}(Q)$, where $Q$ ranges over the bounded orthants contained in $\varphi_{i}^{-1}(U)$, and with the union of the bounded quadrants contained in $\varphi_{i}^{-1}(U)$. Consequently, the union of the images $\varphi_{i}(\operatorname{Int}(Q))$, where $Q$ ranges over the bounded quadrants contained in $\varphi_{i}^{-1}(U)$, coincides with the union of the images

$$
\varphi_{i}\left(\operatorname{dom}_{i}(Q) \cap(-1,1)^{m}\right),
$$

where $Q$ ranges over the bounded orthants contained in $\varphi_{i}^{-1}(U)$.

COROLlary (on rectilinearization of a continuous definable function). Let $U$ be a bounded open subset in $\mathbb{R}^{m}$ and $f: U \rightarrow \mathbb{R}$ be a continuous definable function. Then there exists a finite collection of modifications

$$
\varphi_{i}:[-1,1]^{m} \rightarrow \mathbb{R}^{m}, \quad i=1, \ldots, p,
$$

such that

1) each $\varphi_{i}$ extends to a $\mathcal{W}$-analytic mapping in a neighbourhood of the cube $[-1,1]^{m}$, which is a composite of finitely many local blowings-up with smooth centers and power substitutions;

2) each set $\varphi_{i}^{-1}(U)$ is a finite union of bounded quadrants in $\mathbb{R}^{m}$;

3) each set $\varphi_{i}^{-1}(\partial U)$ is a finite union of bounded closed quadrants in $\mathbb{R}^{m}$ of dimension $m-1$;

4) $U$ is the union of the images $\varphi_{i}\left(\operatorname{dom}_{i}(Q) \cap(-1,1)^{m}\right)$ with $Q$ ranging over the bounded orthants $Q$ contained in $\varphi_{i}^{-1}(U), i=1, \ldots, p$;

5) for every bounded orthant $Q$, the restriction to $\operatorname{dom}_{i}(Q)$ of each function $f \circ \varphi_{i}$ either vanishes or is a normal crossing or a reciprocal normal crossing on $Q$, unless $\varphi_{i}^{-1}(U) \cap Q=\emptyset$.

In a similar way, Theorem $1^{*}$ yields the results stated below. They hold true provided that the real convergent Weierstrass system $\mathcal{W}$ under study is closed under complexification. 
Theorem $2^{*}$ (on rectilinearization of a definable function). Let $U \subset \mathbb{R}^{m}$ be a bounded open subset and $f: U \rightarrow \mathbb{R}$ be a definable function. Then there exists a finite collection of modifications

$$
\varphi_{i}:[-1,1]^{m} \rightarrow \mathbb{R}^{m}, \quad i=1, \ldots, p,
$$

such that

1) each $\varphi_{i}$ extends to a $\mathcal{W}$-analytic mapping in a neighbourhood of the cube $[-1,1]^{m}$, which is a composite of finitely many local blowings-up with smooth centers;

2) each set $\varphi_{i}^{-1}(U)$ is a finite union of bounded quadrants in $\mathbb{R}^{m}$;

3) each set $\varphi_{i}^{-1}(\partial U)$ is a finite union of bounded closed quadrants in $\mathbb{R}^{m}$ of dimension $m-1$;

4) $U$ is the union of the images $\varphi_{i}(\operatorname{Int}(Q))$ with $Q$ ranging over the bounded quadrants contained in $\varphi_{i}^{-1}(U), i=1, \ldots, p$;

5) for every bounded quadrant $Q$, the restriction to $Q$ of each function $f \circ \varphi_{i}$ either vanishes or is a fractional normal crossing or a reciprocal fractional normal crossing on $Q$, unless $\varphi_{i}^{-1}(U) \cap Q=\emptyset$.

COROLlarY* (on rectilinearization of a continuous definable function) $\left({ }^{2}\right)$. Let $U$ be a bounded open subset in $\mathbb{R}^{m}$ and $f: U \rightarrow \mathbb{R}$ be a continuous definable function. Then there exists a finite collection of modifications

$$
\varphi_{i}:[-1,1]^{m} \rightarrow \mathbb{R}^{m}, \quad i=1, \ldots, p,
$$

such that

1) each $\varphi_{i}$ extends to a $\mathcal{W}$-analytic mapping in a neighbourhood of the cube $[-1,1]^{m}$, which is a composite of finitely many local blowings-up with smooth centers;

2) each set $\varphi_{i}^{-1}(U)$ is a finite union of bounded quadrants in $\mathbb{R}^{m}$;

3) each set $\varphi_{i}^{-1}(\partial U)$ is a finite union of bounded closed quadrants in $\mathbb{R}^{m}$ of dimension $m-1$;

4) $U$ is the union of the images $\varphi_{i}\left(\operatorname{dom}_{i}(Q) \cap(-1,1)^{m}\right)$ with $Q$ ranging over the bounded orthants $Q$ contained in $\varphi_{i}^{-1}(U), i=1, \ldots, p$;

5) for every bounded orthant $Q$, the restriction to $\operatorname{dom}_{i}(Q)$ of each function $f \circ \varphi_{i}$ either vanishes or is a fractional normal crossing or a reciprocal fractional normal crossing on $Q$, unless $\varphi_{i}^{-1}(U) \cap Q=\emptyset$.

Theorem $2^{*}$ will be used in the next section and also in our subsequent paper [15]. The significance of the above corollary lies in its application to the theory of arc-analytic functions, presented in our next paper [14]. Finally,

$\left({ }^{2}\right)$ Here the superscript ${ }^{*}$ indicates that the conclusion holds true provided that the real convergent Weierstrass system under consideration is closed under complexification. 
let us mention that the foregoing theorems on rectilinearization imply the classical results of Bierstone-Milman [1] and Parusiński [16].

3. Application to quantifier elimination. As before, we fix a real convergent Weierstrass system $\mathcal{W}$ closed under complexification, and denote by $\mathcal{R}$ the o-minimal structure determined by $\mathcal{W}$. We begin by giving the affirmative answer, for the case of the structure $\mathcal{R}$, to a question posed in our previous paper [1] (Open Problem 1).

TheOREM 3 (on decomposition into special cubes). Let $M$ be a $\mathcal{W}$ analytic manifold. Then every relatively compact $\mathcal{W}$-subanalytic subset $F$ of $M$ is a finite union of special cubes $S_{i}$, i.e. subsets in $M$ of the form

$$
S_{i}=\varphi_{i}\left((-1,1)^{d_{i}}\right)
$$

where each $\varphi_{i}(x)$ is a special modification, i.e. a diffeomorphism from $(-1,1)^{d_{i}}$ onto $S_{i}$ that extends to a $\mathcal{W}$-analytic mapping in the vicinity of $[-1,1]^{d_{i}}$.

Moreover, each $\varphi_{i}$ is a composite of finitely many local blowings-up with smooth centers, and therefore each special cube $S_{i}$ and each inverse mapping

$$
\psi_{i}: S_{i} \rightarrow(-1,1)^{d_{i}}
$$

to the special modification $\varphi_{i}$ is described by terms in the language of restricted $\mathcal{W}$-analytic functions augmented by the name of the reciprocal function.

REMARK. Each inverse mapping $\psi_{j}$ is given piecewise by terms in the language of restricted $\mathcal{W}$-analytic functions augmented by the name of the reciprocal function $1 / x$, because - roughly speaking - it has been locally built in the process of blowing up as a successive superposition of restricted $\mathcal{W}$ analytic functions and of the reciprocal function $1 / x$ off the zero argument.

The proof is by induction with respect to the dimension $m$ of the ambient space $M$; the case $m=0$ is trivial. So take $m>0$, and suppose the assertion holds for all dimensions $<m$. Apply Theorem $2^{*}$ to the function $f:=1-\chi_{F}$, where $\chi_{F}$ is the characteristic function of the set $F$, so as to find a finite collection of modifications

$$
\varphi_{i}:[-1,1]^{m} \rightarrow M, \quad i=1, \ldots, p,
$$

such that

1) each $\varphi_{j}$ extends to a $\mathcal{W}$-analytic mapping in a neighbourhood of the cube $[-1,1]^{m}$, which is a composite of finitely many local blowings-up with smooth centers;

2) $F$ is a finite union of the images under the $\varphi_{j}$ 's of the interiors of some bounded quadrants $Q$.

Then we must show that each $\varphi_{j}(\operatorname{Int}(Q))$ is a finite union of special cubes. We have the following dichotomy: 
either $Q$ is contained in the exceptional divisor $E$ of $\varphi_{j}$, or not.

In the former case, $\varphi_{j}(Q)$ lies in a submanifold of $M$ of dimension $<m$, and thus the assertion holds by induction hypothesis. In the latter, we get

$$
\varphi_{j}(Q)=\varphi_{j}(Q \cap E) \cup \varphi_{j}(Q \backslash E) .
$$

Again, the set $\varphi_{j}(Q \cap E)$ has a decomposition into special cubes by induction hypothesis. Clearly, it is sufficient to decompose the set $Q \backslash E$ into special cubes. But this follows directly from the theorem on decomposition into special cubes from [11, which completes the proof.

We immediately obtain the quantifier elimination result below, which generalizes a theorem of Denef-van den Dries [4] on quantifier elimination for the expansion of the real field by restricted real analytic functions.

COROLlary. The expansion $\mathcal{R}$ of the real field $\mathbb{R}$ admits quantifier elimination in the language of restricted $\mathcal{W}$-analytic functions augmented by the name of the reciprocal function.

Acknowledgements. This research was partially supported by Research Project No. N N201 372336 from the Polish Ministry of Science and Higher Education.

\section{References}

[1] E. Bierstone and P. D. Milman, Arc-analytic functions, Invent. Math. 101 (1990), 411-424.

[2] -, - Canonical desingularization in characteristic zero by blowing up the maximum strata of a local invariant, Invent. Math. 128 (1997), 207-302.

[3] - - - Resolution of singularities in Denjoy-Carleman classes, Selecta Math. (N.S.) 10 (2004), 1-28.

[4] J. Denef and L. van den Dries, p-adic and real subanalytic sets, Ann. of Math. 128 (1988), 79-138.

[5] J. Denef and L. Lipshitz, Ultraproducts and approximation in local rings. II, Math. Ann. 253 (1980), 1-28.

[6] L. van de Dries, On the elementary theory of restricted elementary functions, J. Symbolic Logic 53 (1988), 796-808.

[7] L. van de Dries, A. Macintyre and D. Marker, The elementary theory of restricted analytic fields with exponentiation, Ann. of Math. 140 (1994), 183-205.

[8] H. Hironaka, Introduction to real-analytic sets and real-analytic maps, Istituto Matematico "L. Tonelli", Pisa, 1973.

[9] H. Matsumura, Commutative Algebra, Benjamin/Cummings, Reading, MA, 1980.

[10] K. J. Nowak, A model-theoretic version of the complement theorem, I, Bull. Polish Acad. Sci. Math. 47 (1999), 345-354; II, Applications, 47 (1999), 355-361.

[11] -, Decomposition into special cubes and its application to quasi-subanalytic geometry, Ann. Polon. Math. 96 (2009), 65-74.

[12] -, Quantifier elimination, valuation property and preparation theorem in quasianalytic geometry via transformation to normal crossings, ibid. 96 (2009), 247-282. 
[13] K. J. Nowak, The Abhyankar-Jung theorem for excellent henselian subrings of formal power series, ibid. 98 (2010), 221-229.

[14] -, On arc-analytic functions definable by a Weierstrass system, Ann. Polon. Math., to appear.

[15] -, Gabrielov's rank theorem for differentially algebraic relations, IMUJ Preprint 7, 2009.

[16] A. Parusiński, Subanalytic functions, Trans. Amer. Math. Soc. 344 (1994), 583-595.

[17] J.-P. Rolin, P. Speissegger and A. J. Wilkie, Quasianalytic Denjoy-Carleman classes and o-minimality, J. Amer. Math. Soc. 16 (2003), 751-777.

Krzysztof Jan Nowak

Institute of Mathematics

Jagiellonian University

Łojasiewicza 6

30-348 Kraków, Poland

E-mail:nowak@im.uj.edu.pl

Received 11.2.2009

and in final form 29.7.2009 and 8.4.2010 
